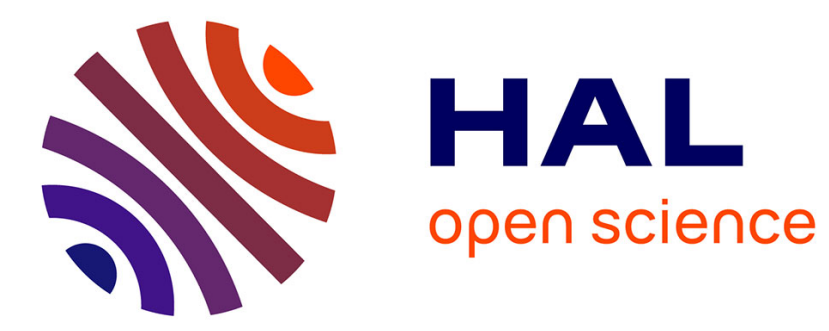

\title{
A stochastic evaluation of the contour strength
}

Fernand Meyer, Jean Stawiaski

\section{To cite this version:}

Fernand Meyer, Jean Stawiaski. A stochastic evaluation of the contour strength. 32nd Annual Symposium of the German Association for Pattern Recognition (DAGM 2010), Sep 2010, Darmstadt, Germany. pp.513-522, 10.1007/978-3-642-15986-2_52 . hal-00614592

\section{HAL Id: hal-00614592 https://hal.science/hal-00614592}

Submitted on 12 Nov 2011

HAL is a multi-disciplinary open access archive for the deposit and dissemination of scientific research documents, whether they are published or not. The documents may come from teaching and research institutions in France or abroad, or from public or private research centers.
L'archive ouverte pluridisciplinaire HAL, est destinée au dépôt et à la diffusion de documents scientifiques de niveau recherche, publiés ou non, émanant des établissements d'enseignement et de recherche français ou étrangers, des laboratoires publics ou privés. 


\title{
A stochastic evaluation of the contour strength
}

\author{
Fernand Meyer ${ }^{1}$ and Jean Stawiaski ${ }^{2}$ \\ ${ }^{1}$ Mines ParisTech - Centre de Morphologie Mathématique - Fontainebleau, France \\ ${ }^{2}$ Philips Healthcare - Medisys Research Laboratory - Paris, France \\ Fernand.Meyer@mines-paristech.fr, Jean.Stawiaski@philips.com
}

\begin{abstract}
If one considers only local neighborhoods for segmenting an image, one gets contours whose strength is often poorly estimated. A method for reevaluating the contour strength by taking into account non local features is presented: one generates a fixed number of random germs which serve as markers for the watershed segmentation. For each new population of markers, another set of contours is generated. "Important" contours are selected more often. The present paper shows that the probability that a contour is selected can be estimated without performing the effective simulations.
\end{abstract}

\section{Introduction}

Image segmentation aims at extracting from an image the contours of the objects of interest. This task is extremely difficult and problem depending. We propose here a stepwise approach, in which we first extract from the image all contours which may be pertinent. These contours are the frontiers of a fine partition of the image. Each tile represents a region which is homogeneous for some criterion. In general these contours are far too numerous and the image is oversegmented. In a second step one orders the contours according to their importance in the image. A dissimilarity between adjacent tiles is estimated. Coarser partitions are then produced by merging all regions whose dissimilarity is below a given threshold ;every time two regions are merged, one reevaluates their dissimilarity with their neighborhood, and the process is stopped as soon some additional criterion is satisfied. Each new fusion produces a finer partition ; these partitions are nested and may be ranked according their coarseness. The weight of a contour is then simply the first level of coarseness where this contour is no more present.

Such nested partitions are called hierarchies. There a two main classes of methods for extracting the contours of interest from the hierarchy, in order to get the final segmentation. In the first a functional is defined and the set of contours maximizing this functional is extracted from the hierarchy $[1,2]$. Another approach to segmentation uses seeds or markers for the regions of interest (including the background). Only the strongest contours separating each pair of markers are retained. For the resulting segmentation to be satisfactory, two conditions are to be met : a) the finest partition should contain all contours of interest ; b) the strength of the contours has to be correctly estimated, otherwise, the wrong contours separating markers will be selected. 
Unfortunately, some contours of objects are intrinsically weak since they correspond to transitions between similar color shades for instance, or objects having almost the same luminance as their background. When such objects are large enough and appear on a clean background, they are nevertheless easily recognized by the human eye, but not easily segmented by the computer. Local methods will estimate these contours as very weak as noticed in [3]. We present in this paper a set of new methods able to produce useful and reliable estimates of the contour strength.

Authors of [4] have described such a method in the context of the watershed segmentation of gradient images. Random germs are spread all over the image and server as markers for the watershed segmentation of a gradient image. Large regions, separated by low contrast gradient from neighboring regions will be sampled more frequently than smaller regions and will be selected more often. On the other hand, high gradient watershed lines will often be selected by the watershed construction, as there are many possible positions of markers which will select them. So the probability of selecting a contour will offer a nice balance between strength of the contours and size of the adjacent regions. The method produces good results for multimedia types of images as well as for 3D granular material but suffers from a major drawback : in order to obtain a robust estimation of the contour strength a relatively large number of simulations has to be made, typically on the order of 50 simulations, implying the construction of 50 watershed. This paper shows how to obtain estimates of the contour strength without simulations. Analyzing under which conditions a given piece of contour will be selected by the watershed makes it possible to compute the contour strength without the need of any simulation. More precisely, one has to determine for each contour the two zones, separated by this contour, where a marker has to be present in order for the contour to be selected.

The paper is organized as follows. In a first section we explain how to produce a hierarchy to start with, obtained with the watershed transform ; we explain why in this case, the strength of the contour is often badly estimated. We then describe an efficient representation of a hierarchy, as a weighted tree. The last part of the paper presents how to implement the stochastic watershed on this tree, in order to estimate a contour strength compatible with the features one desires to stress.

\section{Producing and representing hierarchies}

\subsection{Producing a hierarchy with the watershed transform}

The watershed associates to each regional minimum of a topographic surface its catchment basin. Morphological segmentation applies the watershed transform to the gradient of the image to segment. Like that, one obtains a fine partition where each region is the catchment basin associated to a minimum of the gradient image. 
Suppose now that this same topographic surface is flooded up to some level $\lambda$. A number of lakes are created, some of them are still regional minima of the topographic surface, covering one or more minima of the initial surface ; others are "full", in the sense that the level of the lake reaches the lowest pass point leading to another minimum: such lakes are not regional minima anymore. The catchment basins of this flooded relief form a partition which is coarser than the partition associated to the unflooded surface ; a number of adjacent regions of the fine partition will have merged to form larger regions.

For a level $\mu>\lambda$, the same process can be repeated, leading to a yet coarser partition. Hence, the partitions formed by the catchment basins of a topographic surface, for increasing levels of flooding of this surface, form a hierarchy. This hierarchy can be represented by the fine partition formed by the catchment basins of the unflooded surface plus a dissimilarity measure between adjacent regions, equal to the flooding level for which these regions merge.

\subsection{Dissimilarity associated hierarchies}

More generally, we may associate a hierarchy to each partition for which a dissimilarity between adjacent regions has been defined. Merging all regions with a dissimilarity between some level $\lambda$ produces a coarser partition. For increasing levels $\lambda$ the partitions become coarser and coarser, producing again a hierarchy.

\subsection{Representing a hierarchy as a tree}

In order to give a visual support to the algorithms presented below, we associate to each hierarchy a topographic surface defined as follows. This surface is completely flat, except along the edges of the tile of the finest partition $\Pi$ in the hierarchy. A 0 thickness wall is erected along each piece of contour, with a height equal to the dissimilarity between both adjacent regions. Fig.1 illustrates this construction process. The bottom row shows the successive levels of a hierarchy, where from level to level the most similar regions have merged ; the left most image represents the finest partition. The central row of fig.1 presents the construction of a tree representing the hierarchy. In the left most image, this tree is reduced to isolated nodes, representing each a tile of the partition $\Pi$. Each region $\rho_{i}$ is represented by a node $\nu_{i}$ of the tree, weighted by the area of the region.

We then flood this topographic surface in order to construct a tree representing the hierarchy. The upper row shows the topographic surface during flooding. The left most level shows the walls separating the tiles ; the color of each tile being the color of the source which will flood this tile. As the level of the flood increases, it first reaches the level of the lowest wall : the two adjacent lakes are merged and get a uniform color. As these lakes merge for the first time, an edge is created between the corresponding nodes, with a weight equal to the dissimilarity between both regions ; we write $e_{i j}$ for the edge linking the nodes $\nu_{i}$ and $\nu_{j}$. 


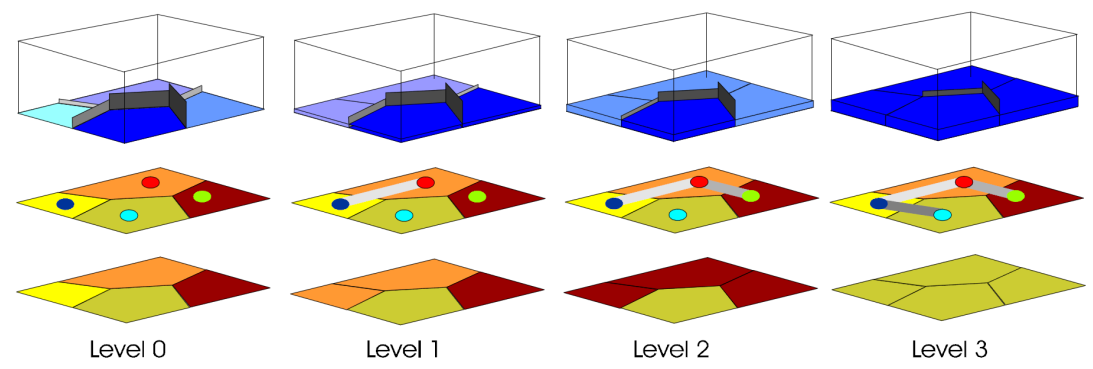

Fig. 1. Construction of the minimum spanning tree by flooding a topographic surface.

At level 2 in fig.1, again two lakes meet for the first time along a wall which separated both lakes and a new edge is created. The same happens at level 3, for which the surface is entirely flooded and the tree which has been constructed spans all nodes. However there exists pieces of walls still emerging from the lakes. As the flood further increases and reaches the level of such a wall, there are not two lakes which meet for the first time, as it is the same lake which is present on both sides of the wall; in this case no edge is created.

In summary, as the flood reaches the level of a wall separating two regions $\rho_{i}$ and $\rho_{j}$ of $\Pi$, there are two possibilities : the lakes on each side of the wall are distinct lakes which merge and an edge $\left(e_{i j}\right)$ is created between the corresponding nodes $\nu_{i}$ and $\nu_{j}$; or both lakes form in reality one and the same lake, created by mergings of lakes at a lower level, in this case, no edge is created between them. This shows that regions may merge at a lower level than the dissimilarity between them : it is sufficient that they belong to a chain of lakes which have merged at lower flooding levels. Based on this remark, we may replace the initial dissimilarity between adjacent regions by the flooding dissimilarity, that is the level for which both regions belong to the same lake during flooding, which is lower (in mathematical terms, this new dissimilarity is a distance, called subdominant ultrametric distance associated to the initial dissimilarity). In Fig.2 we see on the left the initial image and in the center its gradient. The catchment basins of the watershed constitute the finest partition ; the dissimilarity between tiles being the lowest pass point on the gradient image between adjacent regions. The right image shows as dissimilarity the flooding level for which the tiles merge for the first time. The grey tone values on the right are well below the values of the gradient image, specially for large regions, as it is the case for the coat of the cameraman. On the contrary some small contrasted regions are surrounded by extremely bright pieces of contour. This is due to the fact that for larger regions, there are often some parts of the contour which are weaker, leading to early mergings with neighboring regions.

Remark 1. The tree $T$ defined above is the minimum spanning tree of the region neighboring graph defined as follows : each region is represented by a node ; adjacent nodes are linked by an edge with a weight equal to the dissimilarity 


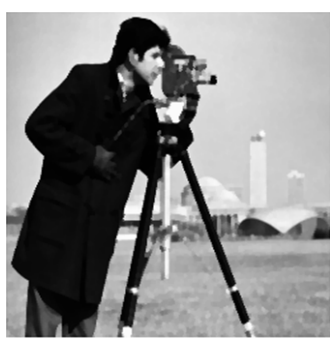

(a)

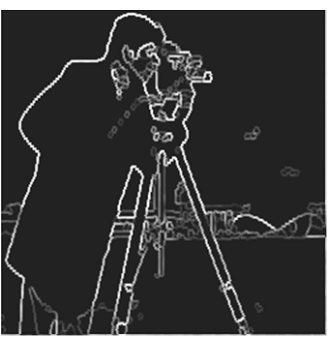

(b)

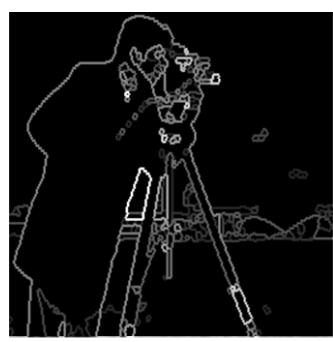

(c)

Fig. 2. (a) Initial image. (b) Gradient value on the contour lines of a segmented image. (c) Contours weighted by the level of flooding for which they disappear.

between both regions. Edges weights along paths of the minimum spanning tree are minimizing the maximum dissimilarity between each pair of nodes [5]. The construction of the flooding tree corresponds also to the single-linkage hierarchy in the context of data clustering.

\subsection{Marker based watershed segmentation}

The flooding tree also plays an important role in the marker based watershed construction. Some regions, called markers, are selected and play the role of sources from which the topographic surface will be flooded. Their flow is such that they create lakes with a uniform and growing altitude. Consider now the evolution of a particular lake $\Lambda_{i}$ when the flooding level increases and reaches the lowest pass point leading to another region. If there is a lake on the other side, there are two possibilities : this lake is a distinct lake $\Lambda_{j}$, produced by another source and these lakes meet for the first time. The pass point where they meet corresponds to an edge of the flooding tree. This edge will be assigned a dissimilarity 1 . If on the contrary the lake on the other side is the same lake $\Lambda_{i}$, originating from the same source, the corresponding edge does not belong to the flooding tree. If the region on the other side is still dry, it will be flooded by the overflood of our lake and they will form a unique lake ; in this case, the corresponding edge belongs to the flooding tree, but does not correspond to a contour of the marker based segmentation : it is assigned a dissimilarity 0 . It is noteworthy that all significant events such as mergings of lakes and overflood of a lake into its neighbor take place along the tree constructed above.

The new binary distribution of weights on the flooding tree represents the marker based segmentation. Cutting all edges with weight 1 produces a forest. Each tree of this forest has its root in a distinct marker. The union of all regions belonging to a same tree of the forest constitutes a region of the marker based segmentation. In mathematical terms, marker based segmentation results in a minimum spanning forest of the neighborhood graph, in which each tree is rooted in a marker [5]. 


\section{Stochastic evaluation of the strength of the contours}

\subsection{Stochastic marker based watershed}

The stochastic evaluation of the contour strength as defined by Angulo et al. [4] assigns to each piece of contour the probability to appear as contour in a watershed segmentation, with random markers. As we have seen, marker based segmentation results in a forest derived from the flooding tree by cutting some edges of this tree. In order to compute the probability that such an edge becomes a contour we have to understand for which marker distribution this happens. We have defined markers as particular regions of the hierarchy which act as sources. One way to select such regions as markers is by drawing random punctual germs onto the surface. If at least one germ falls in a given region, this region is selected as markers ; a region without a germ is not a marker and will be flooded through sources placed in other regions.

Let us first consider two markers $m_{1}$ and $m_{2}$. Both markers become sources which pour water. Their flow is such that they create lakes with a uniform and growing altitude. These two lakes keep separated until they finally meet on the lowest edge, at an altitude $\lambda$ separating them, which becomes a contour edge. Each lake will have covered a number of catchment basins separated by walls with a lower altitude than $\lambda$. Both lakes $L_{1}$ and $L_{2}$ are separated from the rest of the topographic surface by edges higher than $\lambda$.

If one of the regions, for instance $L_{1}$ is without a marker, it can only be flooded through a neighboring region. The lowest connecting edge to a neighboring region is the edge $e_{12}$ of altitude $\lambda$. So if the region $L_{2}$ has a marker, it will flood the region $L_{1}$ through $e_{12}$, which is not selected as a contour edge.

If both regions $L_{1}$ and $L_{2}$ are without markers, then the union of both regions $L_{1} \cup L_{2}$ will be flooded from outside, through a pass point of altitude greater than $\lambda$ and again the edge $e_{12}$ between both regions will not be selected as contour edge.

The same analysis may now be reformulated using the flooding tree. Consider and edge $e_{12}$ with an altitude $\lambda$ of the tree, joining two nodes $\nu_{1}$ and $\nu_{2}$. Cutting all edges of the tree with an altitude higher than or equal to $\lambda$ produces a forest. Let us call $T_{1}$ and $T_{2}$ the trees of the forest containing respectively the nodes $\nu_{1}$ and $\nu_{2}$. The edge $e_{12}$ will be a contour edge in a marker based segmentation, if and only if each of the trees $T_{1}$ and $T_{2}$ has at least one node which is a marker.

The next section will propose several modes for promoting randomly a node to a marker in the tree. For each mode, we then compute the probability of the edges of the tree to become contour edges. 


\subsection{Contour strength for various random distribution of markers}

Uniform Poisson distribution of germs on the surface : the surfacic stochastic watershed. We first use points as germs. The simplest distribution of germs is a Poisson distribution. For a fixed number $N$ of germs this reduces to a uniform distribution of $N$ germs on the topographic surface. As soon one or more germs fall into a region $R_{1}$ of the fine partition $\Pi$, the corresponding node is selected as marker. The probability that a germ falls into a region $A$ of area $\bar{A}$ obeys a binomial distribution of probabilities $p=\frac{\bar{A}}{S}$ and $1-p$, where $S$ is the area of the domain occupied by the function $f$. So the probability that out of $N$ germs no one falls into $A$ is $\left(1-\frac{\bar{A}}{S}\right)^{N}$.

Using the same notations as above, we consider an edge $e_{12}$ of altitude $\lambda$. We cut all edges higher or equal than $\lambda$ and consider the trees $T_{1}$ and $T_{2}$ of the forest adjacent to $e_{12}$. The edge $e_{12}$ is a contour edge in a marker based segmentation, if and only if each of the trees $T_{1}$ and $T_{2}$ adjacent to $e_{12}$ has at least one node which is a marker. This means that at least one germ has fallen in each of the regions $L_{1}$ and $L_{2}$ spanned by both trees. We have to compute the probability of the event $\left\{\right.$ there is at least one germ in $\left.L_{1}\right\}$ and there is at least one germ in $\left.L_{2}\right\}$. The opposite event is the union of two non exclusive events \{there is no germ in $\left.L_{1}\right\}$ or $\left\{\right.$ there is no germ in $\left.L_{2}\right\}$. Its probability is $p$ there is no germ in $\left.L_{1}\right\}+p\left\{\right.$ there is no germ in $\left.L_{2}\right\}-p\left\{\right.$ there is no germ in $\left.L_{1} \cup L_{2}\right\}$, which is:

$$
P_{e_{1,2}}=1-\left(1-\frac{\overline{L_{1}}}{S}\right)^{N}-\left(1-\frac{\overline{L_{2}}}{S}\right)^{N}+\left(1-\frac{\overline{L_{1}}+\overline{L_{2}}}{S}\right)^{N} \text {. }
$$

As we have assigned to each node $\nu_{i}$ of the tree $T$ the area of the underlying region, the area $\overline{L_{1}}$ is simply the sum of the weights of the nodes of the tree $T_{1}$ in the forest obtained after cutting the edges above $\lambda$. We write meas $\left(T_{1}\right)=\overline{L_{1}}$.

The volumic stochastic watershed. In order to give more importance to the contrast an alternative measure may be used: we suppose that the marker is not thrown on the surface but within the volume of the lakes. Considering again the edge $e_{i j}$ of altitude $\lambda$, the adjacent regions spanned by the subtrees $T_{1}$ and $T_{2}$ may be flooded up to level $\lambda$ and will then be covered by two lakes $L_{1}$ and $L_{2}$ with volumes $\left(V_{1}=\lambda \times \operatorname{meas}\left(T_{1}\right)\right)$ and $\left(V_{2}=\lambda \times \operatorname{meas}\left(T_{2}\right)\right)$. If $\Lambda$ is the highest dissimilarity between two regions of the hierarchy, the volume occupied by a lake covering the total surface is $(V=\Lambda \times \operatorname{meas}(T))$. The probability that $e_{i j}$ is selected as an edge is then:

$$
P_{e_{1,2}}=1-\left(1-\frac{V_{1}}{V}\right)^{N}-\left(1-\frac{V_{2}}{V}\right)^{N}+\left(1-\lambda \frac{V_{1}+V_{2}}{V}\right)^{N} .
$$

The surfacic stochastic watershed with non punctual seeds. In what precedes we have imagined punctual seeds, able to fall at any point of both regions $L_{i}$ and $L_{j}$ adjacent to the edge $e_{i j}$. If the seeds are not points, but a set 
$B$, the probabilities will be changed, as the probability that the set $B$ falls within a region $X$ will be proportional, not to the area of $X$, but to the area $\overline{X \ominus B}$ of the set $X$ eroded by the structuring element $B$. This value does depend upon the shape of $X$ and we have to measure it on the initial image.

Mutatis mutandis, the area stochastic watershed will weight the edges with the probability:

$P\left(e_{1,2}\right)=1-\left(1-\frac{\overline{L_{1} \ominus B}}{S}\right)^{N}-\left(1-\frac{\overline{L_{2} \ominus B}}{S}\right)^{N}+\left(1-\frac{\overline{L_{1} \ominus B}+\overline{L_{2} \ominus B}}{S}\right)^{N}$

As previously we observe that this probability becomes 0 if a regions becomes too small for containing the set $B$.

Likewise, the volumic stochastic watershed will weight the edges with the probability:

$$
P\left(e_{1,2}\right)=1-\left(1-\frac{V_{1}^{\prime}}{V}\right)^{N}-\left(1-\frac{V_{2}^{\prime}}{V}\right)^{N}+\left(1-\frac{V_{1}^{\prime}+V_{2}}{V}\right)^{N} .
$$

where $V_{1}^{\prime}=w(e) \times \overline{L_{1} \ominus B}$ and $V_{2}^{\prime}=w(e) \times \overline{L_{2} \ominus B}$.

Results. Fig.3 illustrates the results one obtains for the different methods. Fig.3(a) and (b) show the initial image and the level for which the contours vanish during uniform flooding. Fig.3(c) shows the result of the surfacic stochastic watershed where one uses random points spread over the surface as seeds. If the random points are spread within the volume of the lakes, one obtains Fig.3(d), with large regions and small ones if they are contrasted enough. For balls of a given size taken as random seed, all regions where the ball cannot enter vanish yielding the segmentation of Fig.3(e).

Fig.3(c) and Fig.3(e) compares the stochastic watershed where points are used as markers with the result obtained if one uses disks as seeds. Notice that in this last case, many small regions have vanished, but not all ; some small regions are created as they lie between the contours of larger regions.

Furthermore, the methods presented so far start all with a hierarchy defined by a set of weights on the minimum spanning tree and produce a new set of weights which express a preference for a type of regions compared to others (large or contrasted regions for instance). This homogeneity in the representation of the hierarchies permits to chain the process. Fig.3(f) has been obtained by computing first a surfacic stochastic watershed and on the results compute a stochastic watershed obtained with disks as markers. One sees some difference with Fig.3(e) where only the last stochastic watershed has been used. Figure 4

illustrates some hierarchies of contours obtained on images of the Berkeley Segmentation Dataset [6]. Segmentation results were obtained by thresholding the contour strength map obtained with volumic stochastic watershed with point 
seeds. The Volumic stochastic measures permits to obtain a good trade-off between size and contrast of the object to be detected.

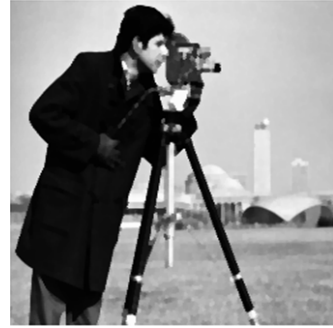

(a)

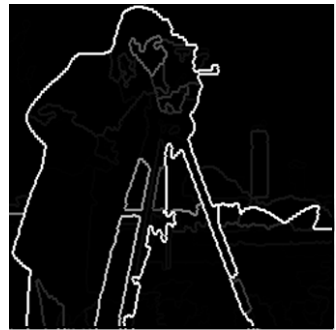

(d)

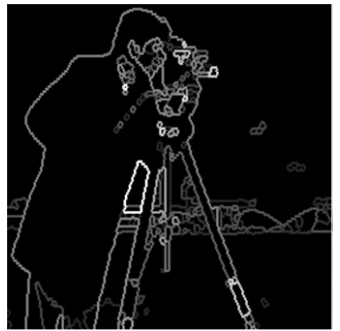

(b)

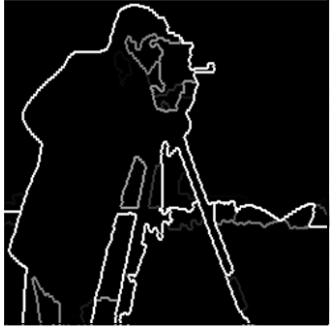

(e)

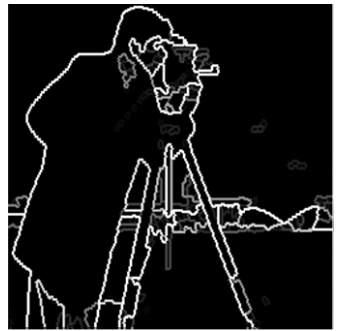

(c)

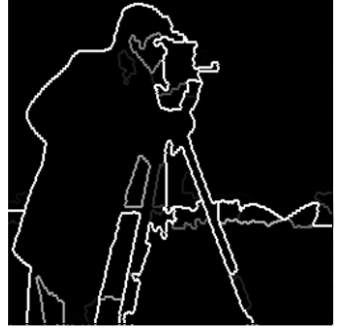

(f)

Fig. 3. (a) Initial image. (b) Flooding level for which the contours disappear. (c) Surfacic stochastic watershed with point seeds. (d) Volumic stochastic watershed with point seeds. (e) Surfacic stochastic watershed with disks as seeds. (f) Stochastic watershed with point seeds followed by a stochastic watershed with disk seeds.

\section{Conclusion}

The stochastic framework is excessively rich as it permits infinite variations : other probability laws for the distribution of seeds, random choice of seeds belonging to a family of shapes, regionalised distribution of seeds according some properties of the domain they will fall [7], etc. An additional nice feature, is that the result always is a probability distribution. That is, whatever the rules which are adopted, the edges get weights between 0 and 1 , which makes it easy to combine hierarchies. Given two hierarchies $H_{1}$ and $H_{2}$ with distinct dissimilarities, it is possible to combine them, by taking for each edge the minimum (resp. maximum) of its dissimilarity in $H_{1}$ and $H_{2}$.

Furthermore, these reevaluation methods of the contours may be chained: use a first distribution of random germs and on the resulting hierarchy, apply a second and then a third, before, ultimately use a marker based segmentation 


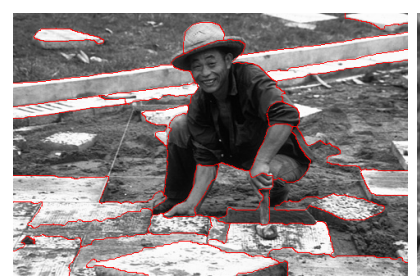

(a)

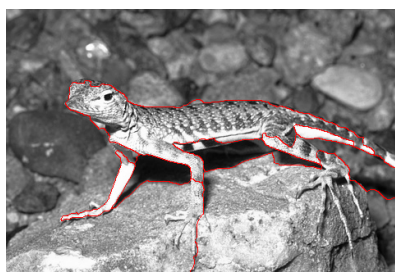

(b)

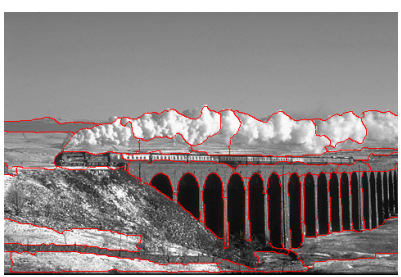

(c)

Fig. 4. Volumic stochastic watershed with point seeds. Segmentation is obtained by thresholding the contour strength.

with markers introduced either in an interactive way or through an automated analysis of the scene.

\section{References}

1. Guigues, L.: Modèles multi-echelles pour la segmentation d'images (in french). These de Doctorat de L'Université de Cergy Pontoise (2003)

2. Guigues, L., Cocquerez, J., Men, H.: Scale-sets image analysis. International Journal of Computer Vision 68(3) (2006) 289-317

3. Najman, L., Schmitt, M.: Geodesic saliency of watershed contours and hierarchical segmentation. IEEE Trans. Pattern Anal. Mach. Intell. 18(12) (1996) 1163-1173

4. Angulo, J., Jeulin, D.: Stochastic watershed segmentation. Int. Symp. Mathematical Morphology ISMM07 (2007) 265-276

5. Meyer, F.: Minimal spanning forests for morphological segmentation. ISMM'94, Mathematical Morphology and its applications to Signal Processing (1994) 77-84

6. Fowlkes, C., Martin, D., Malik, J.: The berkeley segmentation dataset and benchmark (bsdb). www.cs.berkeley.edu/projects/vision/grouping/segbench/

7. Noyel, G., Angulo, J., Jeulin, D.: Classification-driven stochastic watershed. Application to multispectral segmentation. In Proc. of the Fourth European Conference on Color on Graphics, Imaging and Vision (CGIV'2008) (2008) 471-476 